\title{
Limit Models of Pore Space Structure of Porous Materials for Determination of Limit Pore Size Distributions Based on Mercury Intrusion Data
}

\author{
M. Cieszko ${ }^{1}$ (D) $\cdot$ M. Kempiński ${ }^{1}$ (D) T. Czerwiński ${ }^{1}$
}

Received: 30 January 2018 / Accepted: 15 November 2018 / Published online: 28 November 2018

(c) The Author(s) 2018

\begin{abstract}
This paper proposes the application of capillary and chain random models of pore space structure for determination of limit pore diameter distributions of porous materials, based on the mercury intrusion curves. Both distributions determine the range in which the pore diameter distribution of the investigated material occurs and defines the degree of inaccuracy of the method based on the mercury intrusion data caused by the indeterminacy of the sample shape and its pore space architecture. We derived equations describing the quasi-static process of mercury intrusion into the porous layer and porous ball with a random chain pore space structure and analysed the influence of the model parameters on the mercury intrusion curves. It was shown that the distribution of link length in the chain model of the pore space, random location of chain capillaries in the sample and the length distribution of the capillaries do not influence significantly the intrusion process. Therefore, a simple model of the mercury intrusion into the layer is proposed in which chain links of the pore space have random diameters and constant length. This model is used as a basic model of the intrusion process into a sample of any shape and size and with homogeneous and isotropic chain pore space architecture. The thickness of the layer then represents the mean length of chain capillaries in the sample. It was also proved that the capillary and chain models of pore space architecture are limit models of the network model identified in this paper with the pore architecture of the investigated sample. This justifies the application of both models for determination of limit cumulative distributions of pore diameters in porous materials based on the mercury intrusion data.
\end{abstract}

Keywords Mercury porosimetry - Capillary potential curve $\cdot$ Chain and capillary models of pore structure $\cdot$ Limit pore diameter distributions

M. Cieszko

cieszko@ukw.edu.pl

1 Institute of Mechanics and Applied Computer Science, Kazimierz Wielki University, Chodkiewicza 30, 85-064 Bydgoszcz, Poland 


\section{Introduction}

The volume-weighted distribution of pore diameters is the fundamental characteristic of the microscopic pore space structure of porous materials (Scheidegger 1957; Dullien 1979). It defines the volume fraction of pore space points with the assigned pore diameter in the whole pore volume of the sample and makes it possible to assess the basic macroscopic parameters of permeable porous materials, i.e. volume porosity, specific internal surface area, pore area distribution and even permeability and tortuosity (León y León 1998). These parameters play an important role in many physical and chemical processes appearing in such materials, e.g. filtration, transport of mass and energy, wave propagation or chemical reactions.

There are three basic methods used for determination of pore diameter distributions in porous materials: the direct geometrical method based on analysis of 3D microscopic images of the pore space, the indirect method applying the NMR techniques and the method of mercury porosimetry. Geometrical determination of pore diameter distribution consists in assignation to each pore space point (voxel) the maximum diameter of the ball inscribed into the pore space, including this point (Hildebrand and Rüegsegger 1997). It is usually calculated using methods of morphological image analysis (e.g. Shih 2009), and its accuracy is mainly contingent upon resolution of the analysed image. In the case of images created by X-ray computed microtomography, their resolution can achieve the value of $1 \mu \mathrm{m}$. For materials with pores of diameters greater than the image resolution, this method can be used as a "gold standard" (e.g. Arns 2004).

Investigations of porous materials by the NMR techniques consist in measurement of relaxation times of the induced magnetic field of the hydrogen nuclei contained in fluids filling pore space and are based on dependence of this process on quantity, physical properties and the state of fluids in the sample of the material (Coates et al. 1999). This enables direct determination of the presence and quantity of different fluids in the sample, and indirect evaluation of their volume fraction and properties, e.g. viscosity. Determination of pore diameter distributions in porous materials by the NMR loggings has also indirect character. In this case, the linear dependence of the relaxation time on the volume-to-surface ratio of pores filled with fluid containing hydrogen nuclei is used (Brownstein and Tarr 1979; Cohen and Mendelson 1982; Mendelson 1990; Sorland et al. 2007). The range of pore sizes determined in this way depends on the capabilities of the NMR logging for measurements of short relaxation times, and the obtained distributions are qualitatively consistent with that obtained from the microscopic image analysis. However, due to variety of factors influencing the relaxation time, proper interpretation of the NMR logging measurements requires detailed knowledge of the properties of both the skeleton and the fluid in the pore space (Kleinberg and Horsfield 1990; Arns 2004).

Mercury porosimetry is the standard method of experimental determination of the pore diameter distribution in permeable porous materials (León y León 1998; Webb and Orr 1997; Winslow 1984; Giesche 2006). This method applies the non-wetting property of mercury for the surface of almost all materials and consists in determination of the so-called capillary potential curve that relates the volume of mercury intruded into a porous sample with progressively increasing mercury pressure. Interpretation of this curve is based on the assumption that at increasing pressure, mercury is intruded against the capillary forces into the pores of decreasing diameters. This is equivalent to the assumption that the pore structure of the investigated material can be modelled as a bundle of capillaries with random distribution of diameters, crossing the whole sample. In that case, the Washburn formula of menisci equilibrium in a cylindrical capillary can be directly applied for interpretation of the experi- 
mental data. This formula relates the liquid pressure with the diameter of the capillary. As a result, the relation between the relative volume of mercury intruded into the porous sample and the diameter of the capillary is obtained, which is interpreted as a cumulative curve of volume-weighted pore diameter distribution in a sample of the investigated porous material.

The basic advantage of mercury porosimetry, determining its common application for characterization of porous materials, is the wide range of measured pore diameters covering six orders of magnitude, from $3 \mathrm{~nm}$ to $360 \mu \mathrm{m}$. This advantage is also the shortcoming of the method, because it requires application of high pressures that in some cases may deform the measured sample, influencing the obtained results. The obvious shortcoming of mercury porosimetry is the common use of the capillary model of pore space structure of the investigated material for interpretation of mercury intrusion data. This model does not consider the situations that often occur in real porous material, when the large pores are joined with others by narrow necks. This makes it impossible to fill such pores with mercury at the pressure appropriate for their diameter. Therefore, the pore size distribution determined by the capillary model underestimates the volume of large pores, ascribing it to the volume of small pores. In consequence, the obtained distributions may be burdened by significant error. Many authors have also criticized direct application of the Washburn formula for the interpretation of the capillary potential curves.

Many attempts have been made to develop microscopic models of the pore space structure of real porous materials that would include their characteristic spatial connectivity, inhomogeneity and randomness, and would extend the capabilities for modelling processes in which the surface phenomena play an important role. This particularly concerns quasistatic processes of liquid imbibition and drainage. Generally, these models can be divided into analytical and computational models.

The analytical models are mainly those that apply the approach based on percolation theory (Broadbent and Hammersley 1957; Adler 1992; Berkowitz and Balberg 1992; Berkowitz and Ewing 1998) and, in particular, invasion percolation theory (Lenormand and Bories 1980; Wilkinson and Willemsen 1983). In these models, the pore space is represented by the regular network of sites and bonds, and the main emphasis is focused on the analysis of the influence of the geometrical randomness of the medium on the large-scale course of processes in the pore space. A different approach is presented by Chizmadzhev et al. in their monograph (Chizmadzhev et al. 1971), in which the pore space is modelled as a random graph, and the description of the capillary transport is based on the methods of statistical analysis. In this case, a very complex, multi-parametric description is obtained that limits its practical usability.

The computational models can be divided into three types (Bhattad et al. 2011): direct, granular and network. In the direct models, the pore space is reconstructed directly from a 3D microscopic image of a sample of porous material, obtained, for example, by the microcomputer tomography method. In turn, in the granular models the pore space is generated computationally by regular or random packing of simple geometrical elements (e.g. balls) with constant or random sizes representing the skeleton of porous material. In both cases, modelling of processes in the pore space consists in numerical solution of differential equations using the finite element, finite volume or lattice Boltzmann methods. Due to its geometrical and computational complexity, such modelling is time-consuming and can be used only for relatively small samples. This complexity increases even more when processes with surface phenomena are modelled.

An alternative method of pore-scale modelling that substantially increases computational efficiency and reduces the complexity of the local pore geometry, is the approach based on network models of the pore space (Fatt 1956; Blunt 2001; Martins et al. 2009; Bhattad et al. 
2011; Raeesi et al. 2013; Xiong et al. 2016). In these models, the pore space of real materials is represented by a regular or random network of interconnected, geometrically simple elements (e.g. spheres, cuboids or capillaries) of random sizes. Then, the pore description of processes takes the simple form of a Poiseuille-type equation. Considering the complexity and the manner of the construction of the pore network, one can distinguish various generations of models (Bhattad et al. 2011). In the first-generation network models, the pore space is composed of interconnected cylindrical capillaries, whereas in models of the second and third generations, the pore space is represented by a network of large chambers (e.g. spheres and cuboids) interconnected by narrow bonds of various cross sections. In the case of third-generation models, the pore network is created directly from three-dimensional (e.g. microtomographic) pore space images of a sample of porous material using the methods of morphological image analysis. This concerns both the network structure and size distributions of its components. In models of the first and second generations, in turn, the capillary pressure data and porosity are used to tune the numerical network model (Raeesi et al. 2013). This is done using optimization methods by generation of size distributions of the network components that fit the simulated capillary pressure curve and porosity to their experimental values. In this case, the computational complexity of the simulation process is partially transferred to the stage of network generation of the model.

Unlike the microscopic modelling, there are a few proposals for the macroscopic description of the quasi-static processes of non-wetting liquid intrusion into porous materials. The direct application of theories describing liquid transport in unsaturated porous materials to solve this problem, such as the Richards equation (Richards 1931), produces results identical to those for the medium with a capillary pore space structure. This results from the assumption commonly taken in papers in this field that the capillary pressure is a constitutive quantity and is a unique function of saturation with liquid. Such a constitutive assumption causes that the character of the distribution of both quantities in a porous body is always the same. In this case, the homogeneity of the capillary pressure will always induce a homogeneous distribution of saturation with liquid. For this reason, even the advanced thermodynamic models of two-phase flow in porous materials presented in papers by Hassanizadeh and Gray $(1990,1993)$ do not describe the inhomogeneity of liquid distribution during the quasi-static intrusion processes.

A non-standard approach to the problem of macroscopic description of such processes is presented in the paper (Cieszko et al. 2015), where balance equations and constitutive relations have been formulated analysing the process of liquid intrusion in the pressurespace continuum, and menisci motion, as a process of diffusive transport in this continuum. In this model, there are parameters and functions describing pore size distributions in the boundary conditions and in the coefficients characterizing diffusive transport of menisci in the pressure-space continuum. The paper (Cieszko 2016) presents generalization of the macroscopic description of the non-stationary processes of capillary transport of liquid and gas in porous material. It was proved in this paper that such processes take place in the fifth-dimensional pressure-time-space continuum, and quasi-static processes are the special case of this model.

The purpose of the present paper is to formulate a description of mercury intrusion into a porous sample with a relatively simple random chain pore space structure and to propose a method of determining limit pore diameter distributions in porous materials based on the chain and capillary models and the mercury intrusion data.

In the chain model, the individual pores are cylindrical tubes of random length and diameter distributions that are joined at random in a series forming the capillaries of a stepwise changing cross section. The importance of the chain model for interpretation of the mercury intrusion 
data consists in the fact that the chain and capillary models for given pore size distribution and porosity form limit models of the network pore space structure in which cylindrical tubes with random size distribution form a spatial network (Cieszko and Kempiński 2006). The network model reflects the structure of pore space in real porous materials. However, due to the complexity of the description of mercury intrusion into a sample with such a pore structure, formulation of an effective model for interpretation of mercury intrusion data is difficult (Chizmadzhev et al. 1971).

Application of both limit models for interpretation of mercury intrusion data makes it possible to determine limit distributions of pore sizes in the investigated material. Both distributions determine the range in which the pore diameter distribution of the investigated material occurs and defines the degree of inaccuracy of the method caused by the indeterminacy of the sample shape and its pore space architecture. Characterization of pore size distribution in this way is additionally justified by the fact that the accuracy of mercury porosimetry is also limited by at least two other physical assumptions made during interpretation of the mercury intrusion data (León y León 1998): the wetting angle is constant, and the pore space is unchanged during mercury intrusion.

This paper consists of six main sections. Equations describing the quasi-static process of mercury intrusion into porous material with a chain pore structure are derived in the first four main sections. This was done in three stages: first, mercury intrusion into the half-space of porous material with a chain pore space structure was analysed and the integral Volterra equation was obtained for the probability distribution of mercury occurrence in the halfspace. This distribution was used in a section tree to derive the equation for the probability distribution of mercury occurrence in the porous layer during two-side mercury intrusion. Next, an expression describing the saturation of the porous layer with mercury (capillary potential curve) was derived in Sect. 4, and four special cases of the model were obtained. Section 5 shows that the obtained model of mercury intrusion into the porous layer can be used for the description of mercury intrusion into a ball with homogeneous and isotropic chain pore architecture. In this case, particular chains of pores (links) form chords of the ball with random length distribution. The chord length distribution in the ball was used to derive an explicit expression for the ball saturation with mercury.

The obtained results were applied in Sect. 6 to analyse the influence of the model parameters on the capillary potential curve. It was shown that link length distribution in the chain model, location of chain capillaries in the sample and distribution of the capillary length do not influence significantly the capillary potential curve. This proves that a very simple expression describing mercury intrusion into a porous layer with a chain pore space structure and constant link length can be used as a basic description of the capillary potential curve for a porous sample of any shape and size and with chain pore space architecture. The thickness of the layer then represents the mean length of the chain capillaries in the sample.

Section 7 proved that the capillary and chain models of pore space architecture are limit models of the network model identified in this paper with the pore architecture of real porous material. This justifies the application of both models for determination of limit distributions of pore diameters in porous materials, the procedure of which is described in this section. 
(a)

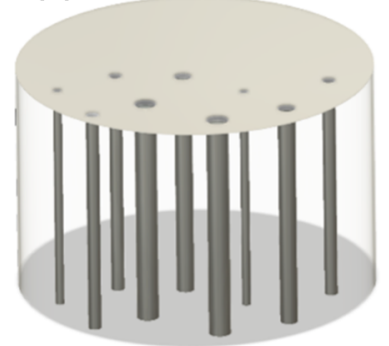

(b)

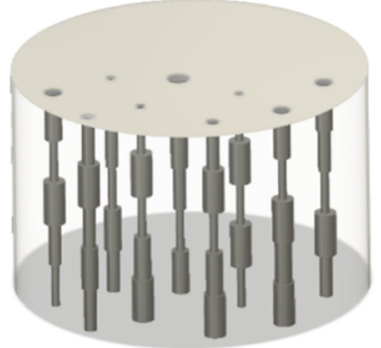

(c)

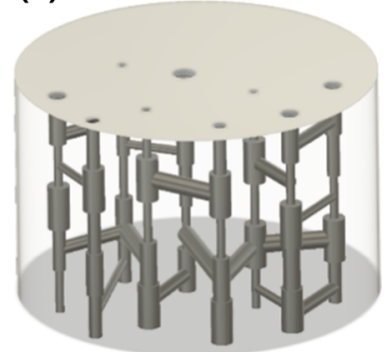

Fig. 1 Schemes of pore space models with capillary (a), chain (b) and network (c) architectures

\section{Modelling of Mercury Intrusion into Porous Half-Space}

\subsection{Basic Assumptions}

We consider the model of porous material in which the individual pores are cylindrical tubes (links) of random diameter $D$ and length $u$ described by the probability distribution $\psi_{o}(D, u)$. Two independent factors determine the pore space structure of such a medium: the pore size distribution $\psi_{o}(D, u)$ and the manner in which they are connected, which we will call here the pore space architecture. A result of the pore architecture is that even for the same pore size distribution in the model material, its pore space structure can be different. Due to the pore architecture, we will distinguish in this paper three kinds of models of the pore space structure: capillary, chain and network (Fig. 1). In the capillary model, links of equal diameter are joined in a series to form long capillaries of constant diameters crossing the whole material. In the chain model, links are randomly joined in series to form capillaries of a stepwise changing cross section. In the network model, randomly connected links form a spatial network.

Considering that mercury does not wet the surface of most materials, under pressure $p$ mercury will enter the porous medium and its menisci will stop on the links of diameter $D$, which is smaller than diameter $D^{*}$ defined by the Washburn expression

$$
D^{*}=-4 \sigma \cos (\theta) / p
$$

where $\sigma$ is the coefficient of the mercury surface tension and $\theta$ stands for the wettability angle of the skeleton material. Relation (2.1) represents the equilibrium condition of the meniscus in a cylindrical tube of diameter $D^{*}$.

Links of a diameter given by condition (2.1), following the paper by Chizmadzhev et al. (1971); we will call here critical. They divide the set of all other links of the model into two classes. The first class consists of the supercritical links of diameters larger than the critical one. These links may be filled with mercury at a given pressure. The other class consists of the subcritical links with diameters smaller than the critical one, which are impossible to fill with mercury at a given pressure. Using the proposed nomenclature, we can say that during the intrusion process into a sample of porous material, mercury fills only the first few supercritical links of the pore space until the first subcritical link occurs. Due to the random character of the link size distribution, the depth of menisci placement in the pore space will take random values. Only in pores on the sample surface with subcritical diameters do the menisci occur on this surface. Consequently, the mercury distribution in a sample of porous material with a chain pore space structure depends on the shape and size of the sample. Only in samples with 
capillary pore architecture is this distribution always homogeneous. In samples with chain pore architecture, each chain capillary is filled with mercury independently of the course of this process in the remaining capillaries. Therefore, the description of mercury intrusion into samples with chain pore architecture is much simpler than such description in samples with network pore architecture.

In this paper, we derive equations describing mercury distribution during the quasi-static process of its intrusion into the half-space, layer and a ball of porous material with chain pore space architecture. These assumptions reduce the complexity of mathematical description of mercury intrusion into porous materials making it possible to derive simple explicit expressions for the mercury intrusion curves. In the analysis we assume, additionally, that distributions of link diameter and length are independent. Then, the link size distribution $\psi_{o}(D, u)$ may be represented as a product of link diameter distribution $\psi(D)$ and link length distribution $\varphi(u)$. We have

$$
\psi_{o}(D, u)=\psi(D) \varphi(u)
$$

In order to ensure the random character of link location in the half-space and layer of porous material, we assume that both media have been cut out from an infinite porous medium with a chain pore space structure by planes perpendicular to the axis of the capillaries. Due to the random location of links in the capillaries, all links on the surfaces of the half-space and the layer will be cut off. Therefore, their length distribution differs from that for links inside the medium.

Taking into account that the condition of the cut-out link occurrence on the surface with the range length $\langle v\rangle=(v, v+d v)$ is the length $u$ of the uncut link to be greater than $v(u \geq v)$, the probability $\eta(v) d v$ of this event will be proportional to the integral

$$
\int_{v}^{\infty} \varphi(u) \mathrm{d} u .
$$

After normalization, the probability distribution $\eta(v)$ of the cut-out link length will be given by

$$
\eta(v)=\frac{1}{\bar{u}} \int_{v}^{\infty} \varphi(u) \mathrm{d} u
$$

where $\bar{u}$ is the average length of (uncut) links in the medium.

Relation (2.3) makes it possible to represent the average length characteristics of the cut-out links by the average length characteristics of the uncut links. We get

$$
\bar{v}=\overline{u^{2}} /(2 \bar{u}), \quad \overline{v^{2}}=\overline{u^{3}} /(3 \bar{u})
$$

\subsection{Description of Mercury Distribution}

We consider a system in which porous material with initially empty pores occupies the halfspace $z>0$ and is in direct contact with the mercury occupying the half-space $z<0$. The pore space architecture of the porous half-space is assumed to be formed of link chains with cut-off boundary links. To derive equations describing the quasi-static process of mercury intrusion into the half-space of porous material, we will first consider a system with uncut boundary links. 
Let the function $F_{O}(z)$ denote the probability of mercury occurrence in any capillary at the depth $z$ of the porous half-space. This function simultaneously defines the probability of mercury occurrence at the depth $z$ in the random chain capillary. We can write

$$
F_{o}(z)=m_{z} / m_{o}
$$

where $m_{o}$ is the mean number of capillaries in the unit area of the surface of porous half-space and $m_{z}$ stands for the number of capillaries in the unit area that are filled by mercury at the depth $z$.

The number $m_{z}$ will be determined considering the set of all capillaries with the first supercritical link. Only in this case will mercury occur inside them. The capillaries filled with mercury at the depth $z$ form a subset of this set.

The number $d m_{u}$ of capillaries in the unit area of the surface of the porous half-space, in which the first link is supercritical and has the range length $\langle u\rangle=(u, u+d u)$, is given by the expression

$$
d m_{u}=m_{o} f_{o}\left(D^{*}\right) \varphi(u) \mathrm{d} u
$$

where

$$
f_{o}\left(D^{*}\right)=\int_{D^{*}}^{\infty} \psi(D) \mathrm{d} D
$$

is the probability of supercritical link occurrence in the medium and $D^{*}$ is given by formula (2.1).

The set of all capillaries with the first supercritical link can be divided into two separate subsets:

- capillaries with the first link of length $u>z$,

- capillaries with the first link of length $u<z$.

In the first case, mercury is present in all capillaries at the depth $z$. The number $m_{z}^{1}$ of these capillaries is given by the expression

$$
m_{z}^{1}=m_{o} f_{o}\left(D^{*}\right) \int_{z}^{\infty} \varphi(u) \mathrm{d} u
$$

In the second case, mercury is present at the depth $z$ when the segment of capillary between the end of the first (supercritical) link and the cross section $z$ is also filled with mercury. Since the probability of such an event is defined by the function $F_{o}(z-u)$, the number $d m_{z}^{2}$ of such capillaries among the capillaries with a first supercritical link and length $u$ can be represented by the formula

$$
\frac{d m_{z}^{2}}{d m_{u}}=F_{o}(z-u) .
$$

Due to relation (2.6), the number $m_{z}^{2}$ of all capillaries of the second type is given by the integral

$$
m_{z}^{2}=m_{o} f_{o} \int_{0}^{z} F_{o}(z-u) \varphi(u) \mathrm{d} u .
$$

Taking into account relations (2.5), (2.8) and (2.10) and that

$$
m_{z}=m_{z}^{1}+m_{z}^{2}
$$


we obtain the integral Volterra equation of the second type,

$$
F_{o}(z)=f_{o} \int_{z}^{\infty} \varphi(u) \mathrm{d} u+f_{o} \int_{0}^{z} F_{o}(z-u) \varphi(u) \mathrm{d} u .
$$

This equation defines the probability $F_{o}(z)$ of mercury occurrence at the depth $z$ of the porous half-space with a chain pore space structure and uncut first links.

Carrying out analogous considerations, the expression for the probability $F(z)$ of mercury occurrence at the depth $z$ of the porous half-space with a chain pore space structure and cut-out first links can be obtained. It takes the form

$$
F(z)=f_{o} \int_{z}^{\infty} \eta(u) \mathrm{d} u+f_{o} \int_{0}^{z} F_{o}(z-u) \eta(u) \mathrm{d} u .
$$

where $\eta(u)$ is the length distribution of the first cut-out links, given by expression (2.3). Function $F_{o}(z)$ in (2.12) is given by Eq. (2.11).

\subsection{Description of Mercury Saturation Distribution}

Using probability distributions $F_{o}(z)$ and $F(z)$ obtained in the previous subsection, we can derive volumetric measures of mercury distributions in both types of porous half-spaces which are directly related to the form of experimental data obtained by the mercury intrusion method, namely saturation of sample with mercury defined as a volume fraction of the pore space occupied by mercury.

Taking into account that layer $\langle z\rangle$ of the porous half-space with the surface area $S$ contains $m_{z} S$ capillaries filled with mercury, the volume $d V$ of mercury in that part of the layer is

$$
d V=m_{z} S d v .
$$

where

$$
d v=\frac{\pi}{4}{\overline{D^{2}}}^{S} d z
$$

is the mean volume of the supercritical links in the layer, while

$$
{\overline{D^{2}}}^{S}=\frac{1}{f_{o}} \int_{D^{*}}^{\infty} D^{2} \psi(D) \mathrm{d} D
$$

is the mean value of their squared diameter. Quantity $\psi(D) / f_{o}$ represents the probability density of the supercritical link diameter distribution.

In turn, the volume $d V_{p}$ of all pores in this part of the layer is given by the formula

$$
d V_{\mathrm{p}}=m_{z} S d v_{o}
$$

where

$$
d v_{\mathrm{o}}=\frac{\pi}{4} \overline{D^{2}} d z
$$

is the average pore volume in the layer $\langle z\rangle$, and

$$
\overline{D^{2}}=\int_{0}^{\infty} D^{2} \psi(D) \mathrm{d} D .
$$

Expressions (2.13) and (2.16) allow one to define saturation $s_{\mathrm{o}}(z)$ of layer $\langle z\rangle$ with mercury. We obtain 


$$
s_{\mathrm{o}}(z)=d V / d V_{p} .
$$

Considering (2.13), (2.16) and (2.5), expression (2.18) takes the form

$$
s_{\mathrm{o}}(z)=F_{\mathrm{o}}(z) f_{2} / f_{o} .
$$

where

$$
f_{2}\left(D^{*}\right)=\int_{D^{*}}^{\infty} \vartheta(D) \mathrm{d} D .
$$

and quantity

$$
\vartheta(D)=D^{2} / \overline{D^{2}} \psi(D) .
$$

can be interpreted as the volumetric distribution of link diameters. It characterizes the volume fraction of links of diameter $D$ in the total pore volume of the medium.

Relation (2.19) makes it possible to transform Eq. (2.11) into the form

$$
s_{o}(z)=f_{2} \int_{z}^{\infty} \varphi(u) \mathrm{d} u+f_{o} \int_{0}^{z} s_{o}(z-u) \varphi(u) \mathrm{d} u .
$$

Similarly, we can transform relation (2.12). We have

$$
s(z)=f_{2} \int_{z}^{\infty} \eta(u) \mathrm{d} u+f_{o} \int_{0}^{z} s_{o}(z-u) \eta(u) \mathrm{d} u
$$

where

$$
s(z)=F(z) f_{2} / f_{o} .
$$

From Eq. (2.22) and relation (2.23), the value of pore saturation with mercury on the half-space surface $(z=0)$ can be obtained,

$$
s_{\mathrm{o}}(0)=\mathrm{s}(0)=f_{2}\left(D^{*}\right) .
$$

\section{Modelling of Mercury Intrusion into Porous Layer}

We apply the probability $F(z)$ describing mercury distribution in the half-space of porous material, given by (2.12), to derive the expression for the probability $G(z)$ of mercury occurrence in a layer of thickness $L$ during the two-side mercury intrusion. Due to the chain architecture of the pore space in the layer, mercury intrusion into pores can be regarded as a two-step process realized first as the left-hand-side intrusion and next as the right-hand-side intrusion or vice versa. During this process, mercury fills the supercritical capillaries which are composed of the supercritical links only, whereas the subcritical capillaries also containing the subcritical links remain partially empty. This means that the left-hand-side and right-hand-side processes of mercury intrusion into subcritical capillaries are independent.

Considering that quantity $F(L)$ defines the probability of mercury occurrence at depth $L$ of the half-space, the number $m_{L}$ of capillaries in a unit area of the half-space surface filled with mercury at depth $L$ is given by the expression

$$
m_{L}=m_{o} F(L) .
$$

where $m_{o}$ is the number of all capillaries in a unit area of the half-space surface. 
Number $m_{L}$, at the same time, represents the number of the supercritical capillaries (fully filled with mercury) in a unit area of the layer surface of thickness $L$ cut out from the halfspace. This means that the number $m^{s}$ of the subcritical capillaries (partially filled with mercury) in a unit area of the layer is equal to the difference of numbers $m_{o}$ and $m_{L}$. We have

$$
m^{s}=m_{o}(1-F(L))
$$

Similarly, the number $m_{z}^{s}$ of subcritical capillaries filled with mercury at depth $z<L$ during the left-hand-side intrusion is equal to the difference of the number $m_{z}$ of all capillaries filled at depth $z$ during this process and the number $m_{L}$ of the supercritical capillaries. We obtain

$$
m_{z}^{s}=m_{o}(F(z)-F(L)) .
$$

Since the processes of the left-hand-side and right-hand-side intrusion of mercury into subcritical capillaries of the layer are independent and equivalent, an expression similar to (3.3) can be formulated for the right-hand-side intrusion of mercury into subcritical capillaries. In this case, the number $m_{L-z}^{s}$ of the subcritical capillaries filled with mercury at depth $s=L-z$ from the right surface of the layer is given by the expression

$$
m_{L-z}^{s}=m_{o}(F(L-z)-F(L)) .
$$

Finally, the number $m_{z}^{T}$ of all capillaries filled with mercury at depth $z$ during its intrusion from both sides into the layer is equal to the sum of the subcritical capillaries filled at this depth and the supercritical one,

$$
m_{z}^{T}=m_{L}+m_{z}^{s}+m_{L-z}^{s}
$$

Taking into account that the probability $F_{L}(z)$ of mercury occurrence in the layer at the depth $z$ can be defined by the formula

$$
F_{L}(z)=m_{z}^{T} / m_{o}
$$

relations (3.2)-(3.5) make it possible to obtain the following expression for the probability $F_{L}(z)$,

$$
F_{L}(z)=F(z)+F(L-z)-F(L) .
$$

Using relation (2.24), from (3.7) we obtain the relation for mercury saturation distribution $s_{L}(z)$ in the porous layer

$$
s_{L}(z)=s(z)+s(L-z)-s(L)
$$

where

$$
s_{L}(z)=F_{L}(z) f_{2} / f_{o} .
$$

Equality (3.8) relates mercury saturation distribution in the porous layer with the mercury distribution in the surface layer of the porous half-space. 


\section{Description of Mercury Intrusion Curve into Porous Layer}

We apply expression (3.8) describing mercury distribution in a porous layer to derive the dependence of the layer saturation with mercury during the intrusion process on the mercury pressure $p$. This dependence is called the capillary potential of porous material and is closely related to the material pore space architecture and its pore size distribution.

The saturation of a porous layer with mercury is defined by the mean value of saturation distribution in the layer. Denoting the capillary potential of the layer by $\bar{s}_{L}(p, L)$, we have

$$
\bar{s}_{L}(p, L)=\frac{1}{L} \int_{0}^{L} s_{L}(z) \mathrm{d} z .
$$

Due to relations (3.8) and (3.9), we obtain

$$
\bar{s}_{L}(p, L)=\bar{F}_{L}(L) f_{2} / f_{o}=(2 \bar{F}(L)-F(L)) f_{2} / f_{o},
$$

where

$$
\bar{F}_{L}(L)=\frac{1}{L} \int_{0}^{L} F_{L}(z) \mathrm{d} z, \quad \bar{F}(L)=\frac{1}{L} \int_{0}^{L} F(z) \mathrm{d} z
$$

are the mean values of the probability of mercury occurrence in the layer and in the surface layer of thickness $L$ of the half-space, respectively.

Considering relation (2.12) between probabilities $F(z)$ and $F_{o}(z)$, and the fact that function $F_{o}(z)$ is given by the integral Eq. (2.11), the capillary potential of the layer (4.2), in general, will not be given in explicit form. The mathematical complexity of the description of this curve for porous material with the chain pore space architecture is caused by the random distribution of link length.

In the next part of this section, we present special models of the chain pore space structure in the porous layer for which the capillary potential (4.2) has an explicit analytical form. We will consider four cases: the capillary model; the chain model with fixed link length and link nodes on the layer surfaces which we will call periodic; the chain model with fixed link length and cut-out surface links which we will call random-periodic; and the chain model with random link length distribution given by the function for which the integral Eq. (2.11) can be solved.

\subsection{Capillary Model}

In the capillary model, the pore space architecture is formed of cylindrical capillaries with a constant cross section and a random distribution of their diameters crossing the whole sample of porous material. In this case, mercury distribution in the layer during the process of mercury intrusion is homogeneous and equal to the value on the layer surface. From Eqs. (2.11) and (2.12), we have

$$
\bar{F}_{L}=\bar{F}=F(0)=F_{o}(0)=F(L)=f_{o}\left(D^{*}\right) .
$$

Then, expression (4.2) reduces to the form

$$
\bar{s}_{L}^{c}(p)=f_{2}\left(D^{*}\right),
$$

where $f_{2}\left(D^{*}\right)$ is given by expression (2.20).

From (4.4), it results that in the model of porous material with capillary pore space architecture, the pore diameter distribution $\vartheta(D)$, given by expression (2.21), completely 
defines the capillary potential curve. This model is commonly used for interpretation of mercury intrusion data.

\subsection{Periodic Chain Model}

The periodic model is the simplest model of chain pore space architecture. Pore space structure in this model is composed of links with fixed lengths and link nodes present on the surfaces of the layer. In this case, the probability distribution $\varphi(u)$ of link lengths takes the form of the Dirac delta function,

$$
\varphi(u)=\delta(u-a),
$$

and the thickness of the layer is a multiple of the link length $a(L=N a)$. Then, the capillary potential curve is described by an expression similar to (4.2),

$$
\bar{s}_{L}^{p}(p, L)=\left(2 \bar{F}_{o}(L)-F_{o}(L)\right) f_{2} / f_{o},
$$

where

$$
\bar{F}_{o}=\frac{1}{L} \int_{0}^{L} F_{o}(z) \mathrm{d} z,
$$

is the mean value of the probability of mercury occurrence in the layer and function $F_{o}(z)$ is given by Eq. (2.11).

Solving Eq. (2.11) for link length distribution (4.5), for example, by the Laplace transformation method, we obtain

$$
F_{o}(z)=1-\left(1-f_{o}\right) \sum_{k=0}^{N} f_{o}^{k} H(z-k a),
$$

where $H(z)$ is the Heaviside step function and $N=L / a$. Then

$$
\bar{F}_{o}=\frac{f_{o}}{N} \frac{1-f_{o}^{N}}{1-f_{o}}, \quad F_{o}(L)=f_{o}^{N} .
$$

Therefore, the capillary potential of the porous layer with periodic pore space architecture, given by expression (4.6), takes the form

$$
\bar{s}_{L}^{p}(p, L)=\left(\frac{2}{N} \frac{1-f_{o}^{N}}{1-f_{o}}-f_{o}^{N-1}\right) f_{2}\left(D^{*}\right) .
$$

Expression (4.9) for $N=1$ takes form (4.4) describing capillary potential curve of porous layer with capillary pore space architecture.

\subsection{Random-Periodic Chain Model}

In the case when the pore space of the layer is composed of link chains with fixed lengths, and the location of these chains is random, the cut-out links will be present on both surfaces of the layer. Due to representation (4.5), the probability distribution $\eta(v)$ of the cut-out link length, defined by expression (2.3), will be uniform,

$$
\eta(v)=H(v) H(a-v) / a .
$$


Determination of the explicit form of the expression describing the capillary potential curve (4.2) requires the solution of integral Eq. (2.11) and relation (2.12) to be used.

Applying solution (4.7), the probability $F(z)$, given by relations (2.12), takes the form of expression

$$
F(z)=1-\left(1-f_{o}\right)\left[1+H(z) \frac{z}{a}-\left(1-f_{o}\right) \frac{1}{a} \sum_{k=0}^{\infty} f_{o}^{k} H(z-k a)(z-k a)\right] .
$$

This function represents the polygonal chain that can be approximated with high accuracy by the smooth function of the form

$$
F_{a}(z)=f_{o}^{z / a+1}
$$

Both functions satisfy the condition: $F(k a)=F_{a}(k a)$, for $k=1,2, \ldots$.

We apply function (4.11) to determine the capillary potential of the layer with randomperiodic pore space architecture. Since

$$
\bar{F}_{o}=\frac{f_{o}}{2 N} \frac{1+f_{o}}{1-f_{o}}\left(1-f_{o}^{N}\right), \quad F(L)=f_{o}^{N+1},
$$

expression (4.2) for the potential curve takes the form

$$
\bar{s}_{L}^{r p}(p, L)=\left(\frac{1}{N} \frac{1+f_{o}}{1-f_{o}}\left(1-f_{o}^{N}\right)-f_{o}^{N}\right) f_{2}\left(D^{*}\right) .
$$

For the case when the number of links in the layer is equal to one $(N=1)$, the randomperiodic model also reduces to the capillary one, despite the presence of one link node in each capillary of the layer. This is caused by homogeneous distribution of the probability $F_{L}(z)$ of mercury occurrence in the layer. In this case: $F_{L}(z)=f_{o}$.

The capillary potential curve determined from the approximated function (4.12) of probability (4.11) is given by the expression

$$
\bar{s}_{L}^{r p}(p, L)=\left(\frac{2}{N} \frac{1-f_{o}^{N}}{\ln \left(1 / f_{o}\right)}-f_{o}^{N}\right) f_{2}\left(D^{*}\right) .
$$

\subsection{Random Chain Model}

To obtain an analytical solution of the integral Eq. (2.11) and the explicit form of the function for the capillary potential of the layer with random chain pore architecture, we assume that the length distribution of links in the model is given by the function

$$
\varphi(u)=\frac{\alpha^{2}-\beta^{2}}{\beta} e^{-\alpha u} \operatorname{sh}(\beta u) .
$$

where $\alpha$ and $\beta$ are parameters of this distribution satisfying the condition $\alpha>\beta$. They define the mean value $\bar{u}$ of link length and their standard deviation $\sigma_{u}$,

$$
\bar{u}=\frac{2 \alpha}{\alpha^{2}-\beta^{2}}, \quad \sigma_{u}^{2}=\frac{2\left(\alpha^{2}+\beta^{2}\right)}{\left(\alpha^{2}-\beta^{2}\right)^{2}} .
$$

Considering that distribution (4.15) contains two parameters, they can be replaced by two basic characteristics of this distribution. We obtain

$$
\alpha=\frac{\bar{u}}{\bar{u}^{2}-\sigma_{u}^{2}}, \quad \beta^{2}=\frac{2 \sigma_{u}^{2}-\bar{u}^{2}}{\left(\bar{u}^{2}-\sigma_{u}^{2}\right)^{2}}
$$


From (4.17), it results that to preserve the positive value of parameters $\alpha$ and $\beta$ characteristics $\bar{u}$ and $\sigma_{u}$ have to satisfy the condition $1>\sigma_{u} / \bar{u}>1 / \sqrt{2}$. Then, the inequality $\alpha>\beta$ is also satisfied.

The solution of the integral Eq. (2.11) obtained by the Laplace transformation method for the link length distribution (4.15) takes the form

$$
F_{o}(z)=f_{o} e^{-\alpha z}\left(\frac{\alpha}{\delta} \operatorname{sh}(\delta z)+\operatorname{ch}(\delta z)\right)
$$

where

$$
\delta=\sqrt{f_{o} \alpha^{2}+\left(1-f_{o}\right) \beta^{2}} .
$$

Then, the probability $F(z)$ defined by expression (2.12) is given by the formula

$$
F(z)=f_{o} e^{-\alpha z}\left(\frac{\alpha^{2}+\delta^{2}}{2 \alpha \delta} \operatorname{sh}(\delta z)+\operatorname{ch}(\delta z)\right) .
$$

Expression (4.20) allows one to determine the quantities $\bar{F}$ and $F(L)$ present in formula (4.2) and to obtain the explicit form of the function $\bar{s}_{L}^{r}(p, L)$ describing the capillary potential curve of the porous layer. From (4.2), we have

$$
\begin{aligned}
\bar{s}_{L}^{r}(p, L)= & \left\{\frac{1}{1-f_{o}} \frac{\bar{u}}{L}\left[\frac{3 \alpha^{2}+\delta^{2}}{2 \alpha^{2}}\left(1-e^{-\alpha L} \operatorname{ch}(\delta L)\right)-\frac{\alpha^{2}+3 \delta^{2}}{2 \alpha \delta} e^{-\alpha L} \operatorname{sh}(\delta L)\right]\right. \\
& \left.-e^{-\alpha L}\left[\frac{\alpha^{2}+\delta^{2}}{2 \alpha \delta} \operatorname{sh}(\delta L)+\operatorname{ch}(\delta L)\right]\right\} f_{2}\left(D^{*}\right)
\end{aligned}
$$

Expressions (4.4), (4.9), (4.14) and (4.21) make it possible to analyse the influence of the link length distribution and their diameters in the chain model of pore space architecture on the capillary potential curve of the porous layer. The differences between these descriptions are defined by the form of the expressions in brackets occurring at the quantity $f_{2}\left(D^{*}\right)$. This quantity defines the saturation of layer surfaces with mercury, regardless of which chain model of pore space architecture is considered. Expressions in brackets, in turn, describe the influence of mercury distribution inside the layer on the capillary potential curve. Detailed analysis of the influence of chain model parameters on this curve is presented in Sect. 6 .

\section{Description of Mercury Intrusion Curve into Porous Ball}

We apply expression (4.2) describing the saturation of the porous layer with mercury during the intrusion process to formulate such a description for mercury intrusion into a porous ball with chain pore space architecture. It is assumed that a spherical sample of porous material has a homogeneous and isotropic architecture of link chains (representing pores) and radius $R$ of the ball is much greater than the mean length $\bar{u}$ of links $(R \gg \bar{u})$. In this case, mercury distribution in the ball is inhomogeneous like in samples with network pore space architecture. However, due to the chain pore architecture, each chain capillary is filled with mercury independently of the course of this process in the rest of the capillaries. Therefore, the description of the intrusion process into particular capillaries of porous ball is the same as the description of mercury intrusion into the porous layer.

Considering the isotropic uniform randomness of straight capillaries in the ball, they have various lengths, the distribution of which can be identified with chord length distribution in the ball. Due to the spherical symmetry of the ball and the homogeneous and isotropic architecture of link chains, the chord length distribution in the ball is equivalent to the distribution of 
segment length cut out by the ball from a homogeneous bundle of parallel straight lines. In this case, the frequency of chord occurrence on the plain perpendicular to the straight lines is homogeneous, and the probability density of chord length distribution $f(L, R)$ is given by linear function of the form (Kellerer 1984),

$$
f(L, R)=\frac{1}{2} \frac{L}{R^{2}},
$$

for $L \in<0,2 R>$. The mean characteristics of this distribution are:

$$
\bar{L}=\frac{4}{3} R, \quad \sigma_{L}=\frac{\sqrt{2}}{3} R .
$$

Distribution (5.1) and expression (4.2) determine the saturation of a porous ball with mercury during intrusion process. To derive the formula describing this saturation, we assume that the porous ball contains $N_{o}$ chain capillaries of different lengths. Then, the number $d N_{o}$ of capillaries in the ball with the range length $\langle L\rangle=\left\langle L, L+d L>\right.$, and the volume $d V_{m}$ of mercury contained in these capillaries are given by the expressions

$$
d N_{o}=f(L, R) N_{o} d L, \quad d V_{m}=\bar{s}_{L}(p, L) V_{c} d N_{o}
$$

where

$$
V_{c}=\pi / 4 \overline{D^{2}} L
$$

is the mean volume of the capillary of length $L$.

Integration of relation (5.3) 2 with respect to the capillary length gives expression for the volume of mercury intruded into the ball,

$$
V_{m}(p, R)=\pi / 4 \overline{D^{2}} N_{o} \int_{0}^{2 R} \bar{s}_{L}(p, L) f(L, R) L \mathrm{~d} L .
$$

Dividing both sides of this expression by the volume of all capillaries in the ball,

$$
V_{p}=N_{o} \pi / 4 \overline{D^{2}} \bar{L},
$$

we obtain expression defining ball saturation with mercury

$$
\bar{s}_{B}(p, R)=\frac{V_{m}(p, R)}{V_{p}}=\frac{1}{\bar{L}} \int_{0}^{2 R} \bar{s}_{L}(p, L) f(L, R) L \mathrm{~d} L .
$$

Expression (5.7) defines the capillary potential curve of a porous ball with chain pore architecture.

\section{Analysis of Influence of Model Parameters on the Mercury Intrusion Curve}

To determine the sensitivity of the capillary potential curve of a porous ball with chain pore space architecture on model parameters, we will analyse the influence of the following factors: link (pore) length and diameter distributions; random location and length distribution of chain capillaries in a sample; sample size. From integral Eq. (2.11) and expression (2.12) result that link length and random location of chain capillaries in the layer strongly influence the complexity of the chain model. Therefore, these two factors also have a qualitative importance for the model applicability, whereas the influence of the other factors is only of a quantitative character. 
In the model proposed in the paper, the description of the ball capillary potential curve with chain pore architecture, given by expression (5.7), is defined by the function describing such curve for the layer, given by expression (4.2). This causes that influence of parameters characterizing random geometry of pores on the ball potential curve is fully represented by the influence of these parameters on the layer potential curve. Therefore, the analysis of influence of these parameters will be carried out on the layer potential curve.

\subsection{Basic Assumptions}

We perform calculations for volume pore diameter distribution $\vartheta(D)$ described by the beta probability distribution defined on the limit range of diameters $D \in<0, D_{o}>$,

$$
\vartheta(D)=\frac{1}{B(m, n)} \frac{1}{D_{o}}\left(\frac{D}{D_{o}}\right)^{m-1}\left(1-\frac{D}{D_{o}}\right)^{n-1}
$$

where $B(x, y)=\Gamma(\mathrm{x}) \Gamma(\mathrm{y}) / \Gamma(\mathrm{x}+\mathrm{y})$ is the beta function, and $\Gamma(\mathrm{x})$ stands for the gamma function. The parameters $m$ and $n(m, n \geq 0)$ specify the shape of the distribution and their mean characteristics,

$$
\bar{D}=D_{o} \frac{m}{m+n}, \quad \sigma_{D}^{2}=D_{o}^{2} \frac{m n}{(m+n)^{2}(m+n+1)} .
$$

Due to relation (2.21), the volume distribution (6.1) of link diameters determines their frequency distribution $\psi(D)$ in the pore space. We have

$$
\psi(D)=\frac{1}{\overline{1 / D^{2}}} \frac{\vartheta(D)}{D^{2}}
$$

where

$$
\overline{1 / D^{2}}=\frac{1}{D_{o}^{2}} \frac{(m+n-1)(m+n-2)}{(m-1)(m-2)} .
$$

Considering that quantities $\sigma_{D}$ and $\overline{1 / D^{2}}$ have to take positive values, from expressions $(6.2)_{2}$ and (6.4) the following conditions result: $m \geq 2$ and $n \geq 0$.

\subsection{Influence of Link Length Distribution and Random Location of Capillaries}

The capillary potential curves of the porous layer for the four special cases of chain pore architectures described in Sect. 4 are presented in Fig. 2. These concern: the capillary model $(C M,(4.4))$, the periodic model (PM, (4.9)), the random-periodic model (RPM, (4.14)) and the random model (RM, (4.21)). To determine the sensitivity of these models on the link length distribution, curves in Fig. 2 were drawn for three different values of mean link length $\bar{u}$ in the random model. Simultaneously, it was assumed that this quantity is equal to the link length $a$ in the periodic and random-periodic models $(\bar{u}=a): N=L / a=10,30,50$. For the remaining parameters of link length and link diameter distributions, the following values were taken:

$$
\alpha \bar{u}=3, \quad \beta \bar{u}=\sqrt{3}, \quad m=10, \quad n=15 .
$$




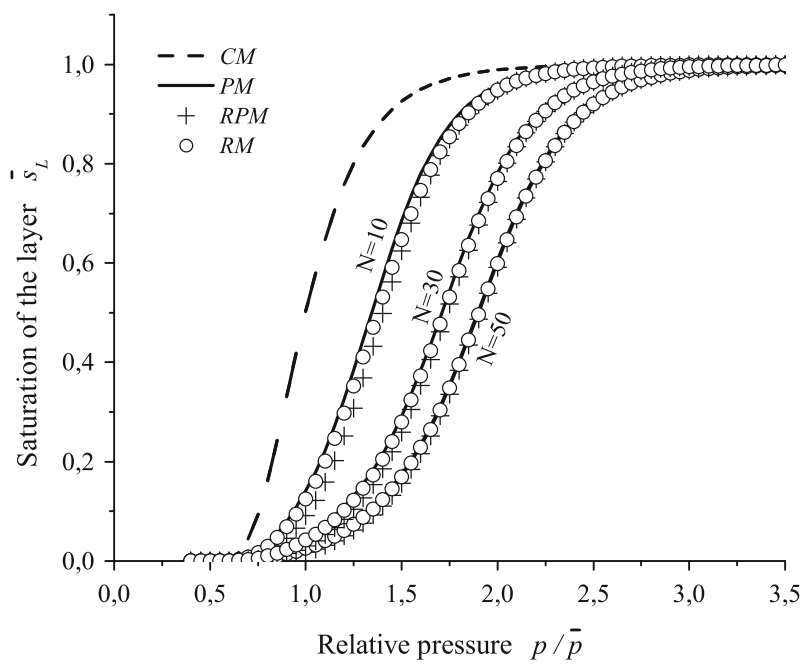

Fig. 2 The capillary potential curves of porous layer with capillary (CM), periodic (PM), random-periodic $(\mathrm{RPM})$ and random $(\mathrm{RM})$ pore space architecture. The curves are drawn for three different values of mean link length in the random model equal to the length of links in the periodic and random-periodic models $(\bar{u}=a)$ : $N=L / a=10,30,50$.

Then, due to relations (4.17) and (6.2), the ratios of characteristics of both distributions take the values:

$$
\left(\sigma_{u} / \bar{u}\right)^{2}=2 / 5, \quad\left(\sigma_{D} / \bar{D}\right)^{2}=3 / 52
$$

This means that the capillary potential curves drawn in Fig. 2 for different mean length $\bar{u}$ of links have also different standard deviations $\sigma_{u}$.

The quantity $\bar{p}$ in Fig. 2 represents mercury pressure for which its menisci are in equilibrium in the cylindrical capillary of diameter equal to the mean diameter $\bar{D}$ of links in the layer. Due to the Washburn relation (2.1), it is given by the expression

$$
\bar{p}=-4 \sigma \cos (\theta) / \bar{D} \text {. }
$$

In Fig. 2, it results that the mercury intrusion curves of a porous layer with the periodic $(P M)$, random-periodic $(\mathrm{RPM})$ and random $(\mathrm{RM})$ pore space architecture are very close for each values of ratio $N=L / \bar{u}=L / a$ and the difference between them increases when this ratio decreases. In the case, when $N \rightarrow 1$, it can be shown that the mercury intrusion curve for the random model considerably differs from those for the capillary, periodic and random-periodic models which take the same form described by expression (4.4) for the capillary pore architecture. This means that link length distribution and random location of chain capillaries in the layer (or cut-out link length distribution on surface of the layer) do not significantly influence the capillary potential curve when the thickness $L$ of the layer is much greater than the mean link length $\bar{u}(N=L / \bar{u}=L / a \gg 1)$. Such condition ensures full statistical representation of link chains (pores) in the layer causing that saturation of the layer with mercury for a given pressure depends mainly on the mean placement of menisci in the layer. This placement is fully determined by the mean link length and probability of supercritical link occurrence in the layer defined by formula (2.7).

From the above analysis, it results that the description of the capillary potential curve of a porous layer with random chain pore architecture can be effectively represented by the 
simple model of constant link length, given by expression (4.9). Therefore, further analysis of the influence of model parameters on the capillary potential curve will be based on the periodic model.

\subsection{Influence of Capillary Length Distribution}

The minor influence of link length distribution and the random location of chain capillaries in the porous layer on its mercury intrusion curve make it possible to reduce the complexity of description of the intrusion curve into a porous ball with chain pore space architecture. Introducing relation (4.9) into formula (5.7), after integration, we obtain

$$
\begin{aligned}
\bar{s}_{B}(p, R)= & \frac{3}{M}\left\{\frac{1}{1-f_{o}}\left[1-\frac{2}{\ln \left(f_{o}^{M}\right)}\left(f_{o}^{M}+\frac{1-f_{o}^{M}}{\ln \left(f_{o}^{M}\right)}\right)\right]\right. \\
& \left.-\frac{1}{f_{o} \ln \left(f_{o}\right)}\left[f_{o}^{M}-\frac{2}{\ln \left(f_{o}^{M}\right)}\left(f_{o}^{M}+\frac{1-f_{o}^{M}}{\ln \left(f_{o}^{M}\right)}\right)\right]\right\} f_{2}\left(D^{*}\right)
\end{aligned}
$$

where $M=2 R / a$. Due to relation (5.2) , we have $M=3 / 2 N$, where $N=\bar{L} / a$.

It can be shown that expression (6.6) satisfies the condition

$$
\lim _{p \rightarrow \infty} \bar{s}_{B}(p, R)=\lim _{f_{o} \rightarrow 1} \bar{s}_{B}(p, R)=1 .
$$

To investigate the influence of the capillary length distribution on the mercury intrusion curve, we compare the model of mercury intrusion into a porous ball, given by expression (6.6), with the model of mercury intrusion into the porous layer, given by (4.9). We assume that the parameters characterizing link diameter distributions in both models are the same and the thickness $L$ of porous layer is equal to the mean length $\bar{L}$ of capillaries (chords) in the ball: $M=L / a=\bar{L} / a$. Both capillary potential curves drawn for the above conditions are presented in Fig. 3. The course of curves in this figure is similar to that presented in Fig. 2. The mercury intrusion curve into a porous ball is very close to the intrusion curve into a porous layer.

Similarly, for $M \rightarrow 1$, it can be shown that the mercury intrusion curve for the porous ball, given by expression (6.6), considerably differs from that for the porous layer, described by expression (4.9). However, when the number $M$ of links in the mean length of capillaries increases, the influence of their length distributions on the capillary potential curve of the ball decreases and both curves approach to each other. In this case, expression (6.6) describing the mercury intrusion curve into a porous ball can be effectively replaced by the simple expression (4.9) for the intrusion curve into the layer. Then, the thickness of the layer $L$, present in expression (4.9), should be interpreted as the mean value of capillary length in the ball.

Generalizing the above considerations, we can state that expression (4.9) can be interpreted as an effective model of mercury intrusion curve into a porous sample of any shape and size when its pore structure is chain, isotropic and homogeneous. In this case, the quantity $L$ in this model represents the mean length of chain capillaries (chords) in the sample. This quantity is the mean characteristics of the shape and size of the sample. For ball-shaped samples, the mean length $\bar{L}$ of chain capillaries is related to the ball radius $R$ by $(5.2)_{1}$. 


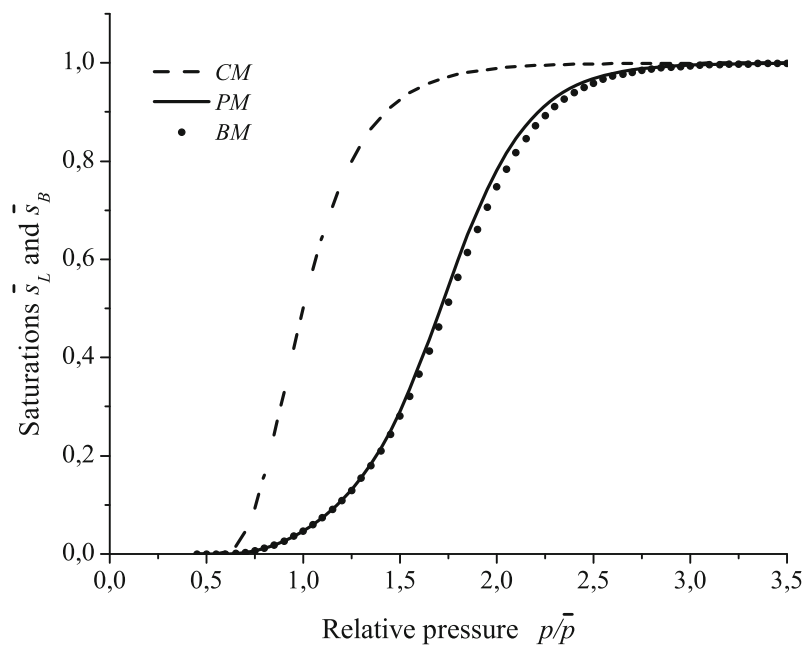

Fig. 3 The capillary potential curves of porous layer and porous ball drawn for thickness $L$ of porous layer equal to the mean length $\bar{L}$ of capillaries (chords) in the ball

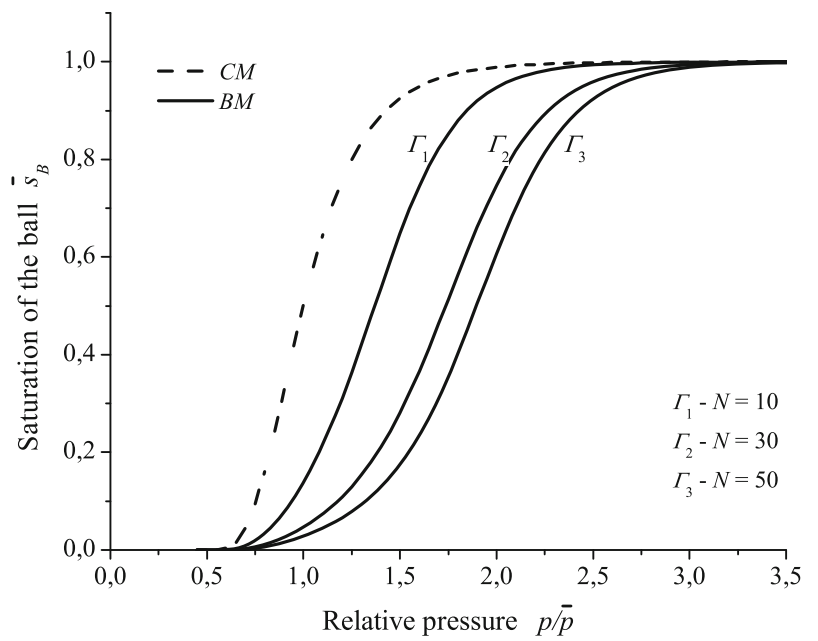

Fig. 4 Influence of the ball radius $R$ and the mean link length $\bar{u}=a, N=\bar{L} / a=4 R / 3 a=2 M / 3$ on the capillary potential curve

\subsection{Influence of other Model Parameters}

The influences of ball radius $R$ (or mean length $\bar{L}$ of chain capillaries in the ball), mean link length $\bar{u}=a$ and parameters $m$ and $n$ of link diameter distribution on the capillary potential curve are presented in Figs. 4 and 5. These figures were drawn based on the model given by relation (4.9) and for the same values of constant parameters used for calculation of curves in Figs. 2 and 3.

Curves in Fig. 4, due to relation $N=\bar{L} / a=4 R / 3 a=2 M / 3$, illustrate simultaneously the influence of both parameters $R$ ( or $\bar{L}$ ) and $a$ on the capillary potential curve. It is visible 


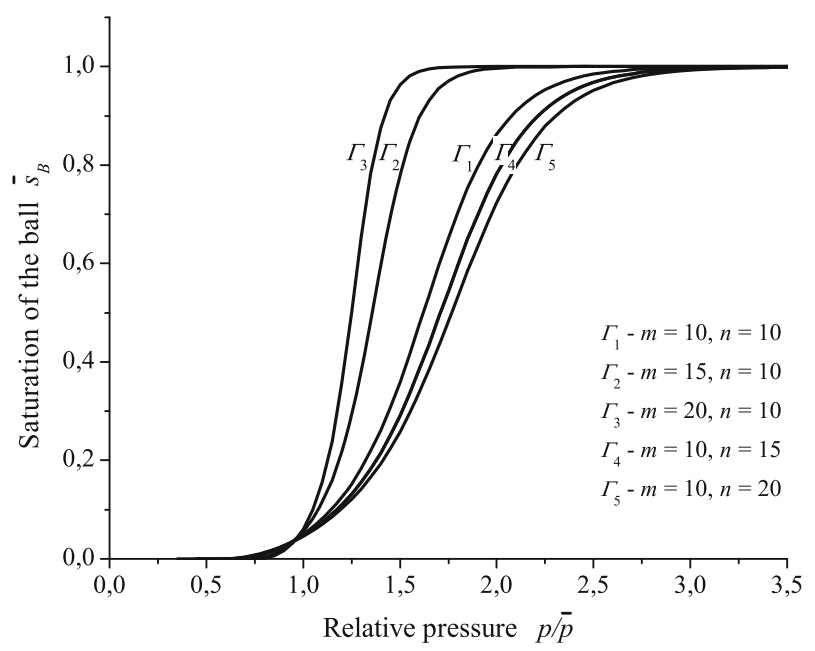

Fig. 5 Influence of shape parameters $m$ and $n$ of link diameter distribution on the capillary potential curve

that an increase in the ball radius (or decrease in the link length) results in transition of the curve to higher pressure values.

The influence of shape parameters $m$ and $n$ of link diameter distribution is shown in Fig. 5 for three various values of these parameters. From this figure, it results that parameters $m$ and $n$ also play the role of shape parameters for the capillary potential curves.

Considering that for the constant shape parameters $m$ and $n$ of link diameter distribution there is a unique linear relation between maximum pore diameter $D_{o}$ and the mean link diameter $\bar{D}$, given by relation $(6.2)_{1}$, the influence of diameter $\bar{D}$ on the capillary potential curve reduces to the rescaling of the pressure coordinate in Figs. 2, 3, 4 and 5. This because expression (4.9) describing the capillary potential curve, written as a function of dimensionless pressure $p / \bar{p}$, does not depend explicitly on the mean diameter $\bar{D}$.

\section{Determination of Limit Pore Diameter Distributions}

In this section, we show that the capillary potential curves of porous samples with capillary and chain pore space architecture are limit curves for samples with any network pore architecture. This proves that both models of the pore space are limit models of the network one with respect to the capillary potential curve. We also propose the use of the capillary and chain pore space architectures for determination of limit cumulative distributions of pore diameters in real porous materials, based on the mercury intrusion data. Both distributions define the range in which the cumulative distribution of pore diameter of the investigated material occurs.

\subsection{Limit Models of Mercury Intrusion Curve}

To provide generality of the analysis, we consider a set of cylindrical tubes (links) of random diameter and length described by a probability distribution. These links are used to form three kinds of porous media with the same porosity and statistically homogeneous and isotropic pore space structures and various pore architectures: capillary, chain and network. They have 


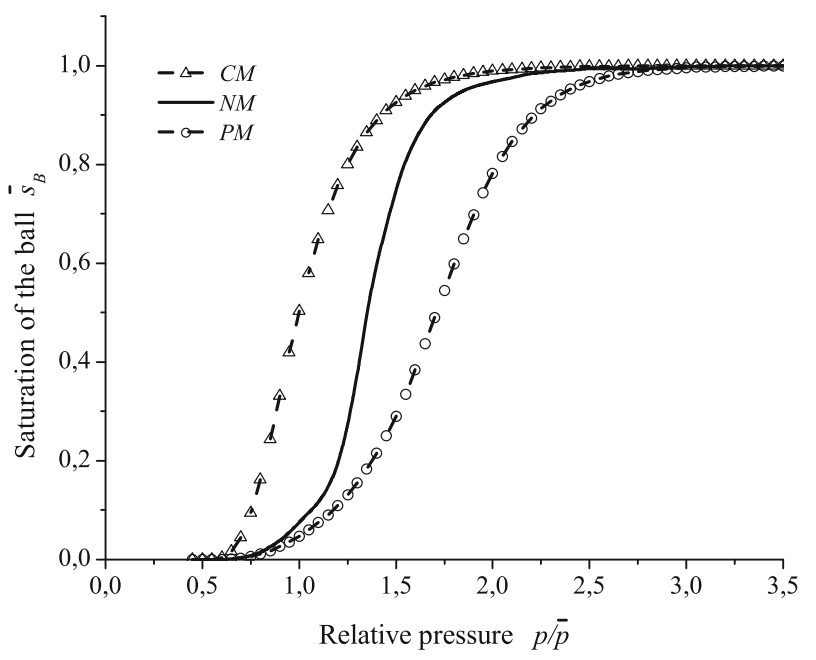

Fig. 6 Mercury intrusion curves into porous ball with capillary (CM), chain (PM) and network (NM) pore space architectures and the same porosity and pore size distribution

the same pore size distribution. A sample of the same shape (e.g. a ball) is cut out from each of these media and subjected to the process of mercury intrusion. Due to various pore space architectures, they are characterized by different mercury intrusion curves (capillary potential curves).

The volume of mercury intruded into a porous sample with capillary pore space architecture is always greater than the volume of mercury intruded into the sample with chain architecture. This is caused by the fact that in the sample with capillary pore architecture all supercritical links are filled with mercury at a given pressure. By comparison, in the sample with chain pore architecture the number of supercritical links filled with mercury is minimal, due to the lack of connections between the chains of links. The occurrence of such connections in the porous sample changes the chain pore architecture into the network one and causes an increase in the number of supercritical links filled with mercury at a given pressure. Consequently, all intrusion curves of porous samples with any network pore space architecture have to lie between the intrusion curves for samples with the capillary and chain architecture (Fig. 6). This means that for a given porosity and link size distribution in a porous sample, the points of the intrusion curves for all possible pore space architectures belong to a set of the band form, limited by the intrusion curves for samples with the capillary and chain pore architecture. In this sense, the capillary and chain models of pore space architecture are two limit cases of the network models.

\subsection{Limit Pore Diameter Distributions}

For these considerations, we assume that the network model of pore space architecture composed of cylindrical tubes is a good representation of the pore space of real porous materials. In this case, determination of the pore diameter distribution in a sample of porous material 


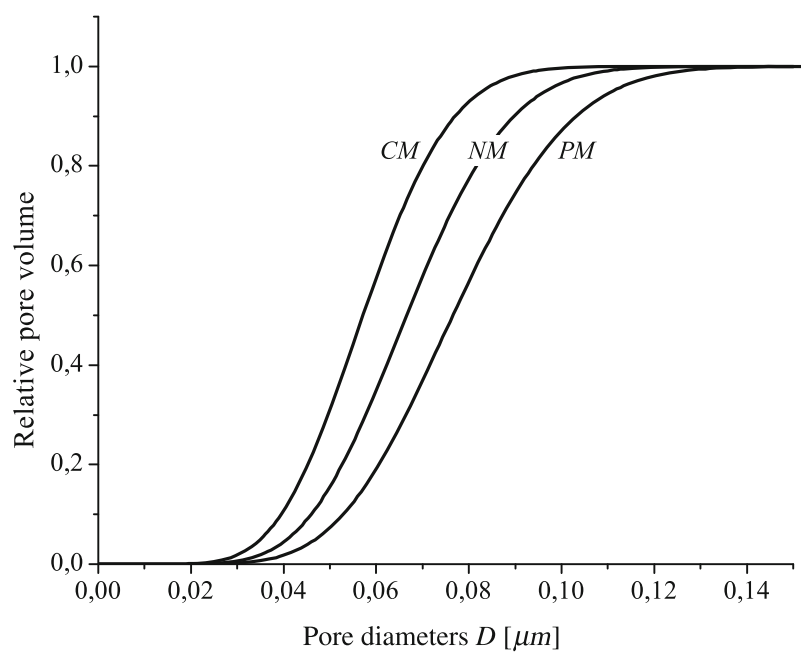

Fig. 7 Cumulative distributions of pore diameters determined by the network (NM), capillary (CM) and chain (PM) models of pore space architecture

based on the mercury intrusion data could be performed as long as the mathematical model of the capillary potential curve for the sample of porous material with the network pore space architecture was available. However, formulation of such an effective model is a very complicated problem (Chizmadzhev et al. 1971).

In this paper, we apply simple limit models of the mercury intrusion curves to propose the procedure for determination of limit cumulative distributions of pore diameters based on the mercury intrusion curves, defining the range of occurrence of cumulative distribution in the investigated material.

The assumption of the network representation of pore space in real porous materials allows one to consider the experimental curve of mercury intrusion as the curve $N M$ in Fig. 6. It lies between the limit curves $C M$ and $P M$ that characterize mercury intrusion into a porous sample of the same shape, porosity and pore size distribution as in the investigated sample, but with capillary and chain pore space architectures. The cumulative distribution of pore diameters in the investigated sample is assumed to be represented by the curve $N M$ shown in Fig. 7. This curve will then also characterize the distribution of link diameters in samples with capillary and chain pore architectures, the capillary potentials of which are given by curves $C M$ and $P M$ in Fig. 6. In this case, interpretation of the experimental curve $N M$ in Fig. 6 by the capillary model, which is given by relation (4.4), is equivalent to the change parameters of the pore diameter distribution. This results in transition of the intrusion curve $C M$ in this figure for the capillary model in the direction of increasing pressures up to cover with the curve $N M$. Such a change of distribution parameters, due to the inverse relationship between pressure and pore diameter given by the Washburn relation (2.1), means transition of the curve $N M$ in Fig. 7 in the direction of decreasing diameters. It takes the form of the curve $C M$ in this figure.

Similarly, interpretation of the experimental curve $N M$ in Fig. 6 by the chain model, given by relation (4.9), is equivalent to the change of parameters of the pore diameter distribution (for the fixed value of the ratio $L / a$ ). This results in transition of the intrusion curve $P M$ for the chain model in the direction of decreasing pressures up to cover with the curve NM. This means transition of the curve $N M$ in Fig. 7 in the direction of increasing diameters. It takes 
the form of the curve $P M$ in this figure. The values of parameters $L$ and $a$ present in the chain model can be estimated based on the volume of the investigated sample and the mean diameter $\bar{D}$, respectively. Both curves: $C M$ and $P M$ in Fig. 7 define the range of occurrence of the cumulative distribution of pore diameters in the investigated material. This range defines the degree of inaccuracy of the method based on the mercury intrusion data caused by the indeterminacy of the sample shape and its pore space architecture.

\section{Final Remarks}

In this paper, we considered stochastic models of porous materials with a chain pore space structure composed of cylindrical tubes (links) with random length and diameter distributions and joined at random in series forming the capillaries of a stepwise changing cross section. The integral Volterra equation describing the quasi-static process of mercury intrusion into the half-space of such material was derived, and the obtained results were used for description of the mercury intrusion curve into a layer and a ball of porous material. Solution of the equation made it possible to obtain analytical expressions for capillary potential curves of porous samples with different chain pore space architectures (capillary, periodic, randomperiodic and random). We have shown that link length distribution, the random location of chain capillaries in the sample and distribution of capillary length do not significantly influence the capillary potential curve. This justifies the use of a simple expression for the capillary potential curve of porous layer with the periodic chain pore architecture as an effective model of mercury intrusion curve into porous sample of any shape and sizes. The thickness of the layer in this model then represents the mean length of chain capillaries in the sample.

It was shown, moreover, that the capillary and chain pore space architectures of porous materials are limit models for network pore architecture with respect to the capillary potential curve. This proves that both models of the pore space are limit models of the network model. We propose application of both limit models for determination of limit cumulative distributions of pore diameters in porous materials investigated by the mercury intrusion method. Both distributions define the range in which the pore diameter distribution of the investigated material occurs. This range defines the degree of inaccuracy of the method based on the mercury intrusion data caused by the indeterminacy of the sample shape and its pore space architecture.

Characterization of pore size distribution in porous materials in this manner is additionally justified by the fact that the accuracy of the mercury porosimetry is also limited by at least two physical assumptions commonly taken during interpretation of the mercury intrusion data: the wetting angle is constant, and the pore space is unchanged during the mercury intrusion.

Considering that interpretation of mercury intrusion data in standard mercury porosimetry is based on the capillary model of pore space architecture and determines the lower limit of pore diameter distribution, the procedure proposed in this paper supplements the mercury porosimetry by also allowing determination of the upper limit distribution estimating the accuracy of this method. 
Open Access This article is distributed under the terms of the Creative Commons Attribution 4.0 International License (http://creativecommons.org/licenses/by/4.0/), which permits unrestricted use, distribution, and reproduction in any medium, provided you give appropriate credit to the original author(s) and the source, provide a link to the Creative Commons license, and indicate if changes were made.

\section{References}

Adler, P.M.: Porous Media. Geometry and Transports. Butterworth-Heinemann, Boston (1992)

Arns, C.H.: A comparison of pore size distributions derived by NMR and X-ray-CT techniques. Physica A 339, 159-165 (2004)

Berkowitz, B., Balberg, I.: Percolation approach to the problem of hydraulic conductivity in porous media. Transp. Porous Med. 9(3), 275-286 (1992)

Berkowitz, B., Ewing, R.P.: Percolation theory and network modeling applications in soil physics. Surv. Geophys. 19(1), 23-72 (1998)

Bhattad, P., Willson, C.S., Thompson, K.E.: Effect of network structure on characterization and flow modeling using X-ray micro-tomography images of granular and fibrous porous media. Transp. Porous Med. 90, 363-391 (2011)

Blunt, M.J.: Flow in porous media: pore-network models and multiphase flow. Curr. Opin. Colloid Interface Sci 6, 197-207 (2001)

Broadbent, S.R., Hammersley, J.M.: Percolation processes I: crystals and mazes. Proc. Camb. Philos. Soc. 53, 629-641 (1957). https://doi.org/10.1017/S0305004100032680

Brownstein, K.R., Tarr, C.E.: Importance of classical diffusion in NMR studies of water in biological cells. Phys. Rev. A 19, 2446 (1979)

Cieszko, M.: Macroscopic description of capillary transport of liquid and gas in unsaturated porous materials. Meccanica 51(10), 2331-2352 (2016)

Cieszko, M., Kempiński, M.: Determination of limit pore size distributions of porous materials from mercury intrusion curves. Eng. Trans. 54(2), 143-158 (2006)

Cieszko, M., Chaplya, Y., Kempiński, M.: Continuum description of quasi-static intrusion of non-wetting liquid into a porous body. Contin. Mech. Therm. 27(1-2), 133-144 (2015)

Chizmadzhev, YuA, Markin, V.S., Tarasevich, M.R., Chirkov, YuG: Macrokinetics of Processes in Porous Media. Nauka, Moscow (1971). (in Russian)

Coates, G.R., Xiao, L., Prammer, M.G.: NMR Logging. Principles and Applications. Halliburton Energy Services, Houston (1999)

Cohen, M.H., Mendelson, K.S.: Nuclear magnetic relaxation and the internal geometry of sedimentary rocks. J. Appl. Phys. 53, 1127 (1982)

Dullien, F.A.L.: Porous Media: Fluid Transport and Pore Structure. Academic Press, New York (1979)

Fatt, I.: The network model of porous media: I. Capillary pressure characteristics. Pet. Trans. AIME 207, 144-159 (1956)

Giesche, H.: Mercury porosimetry: a general (practical) overview. Part. Part. Syst. Charact. 23(1), 9-19 (2006)

Hassanizadeh, S.M., Gray, W.G.: Mechanics and thermodynamics of multiphase flow in porous media including interphase boundaries. Adv. Water Resour. 13, 169-186 (1990)

Hassanizadeh, S.M., Gray, W.G.: Thermodynamic basis of capillary pressure in porous media. Water Resour. Res. 29(10), 3389-3405 (1993)

Hildebrand, T., Rüegsegger, P.: A new method for the model-independent assessment of thickness in threedimensional images. J. Microsc. 185(1), 67-75 (1997)

Kellerer, A.M.: Chord-length distributions and related quantities for spheroids. Radiat. Res. 98, 425-437 (1984)

Kleinberg, R.L., Horsfield, M.A.: Transverse relaxation processes in porous sedimentary rock. J. Magn. Reson. 88(1), 9-19 (1990)

Lenormand, R., Bories, S.: Description d'un mécanisme de connexion de liaison destine à l'étude du drainage avec piégeage en milieu poreux. C. R. Acad. Sci. Paris 291B, 279-283 (1980)

León y León, C.A.: New perspectives in mercury porosimetry. Adv. Colloid Interfaces 76-77, 341-372 (1998)

Martins, A.A., Laranjeira, P.E., Braga, C.H., Mata, T.M.: Modeling of transport phenomena in porous media using network models. Prog. Porous Media Res. 5, 165-261 (2009)

Mendelson, K.S.: Percolation model of nuclear magnetic relaxation in porous media. Phys. Rev. B 41, 562 (1990)

Raeesi, B., Morrow, N., Mason, G. (2013) Pore network modeling of experimental capillary pressure hysteresis relationships. SCA2013-015 (2013) 
Richards, L.A.: Capillary conduction of liquids through porous mediums. J. Appl. Phys. 1, 318 (1931)

Scheidegger, A.E.: The Physics of Flow Through Porous Media. University Press, Toronto (1957)

Shih, F.Y.: Image Processing and Mathematical Morphology. Fundamentals and Applications. Taylor and Francis Group, Boca Raton (2009)

Sorland, G.H., Djurhuus, K., Wideroe, H.C., Lien, J.R., Skauge, A.: Absolute pore size distribution from NMR. Diffus. Fundam. 5, 4.1-4.15 (2007)

Webb, P.A., Orr, C.: Analytical Methods in Fine Particle Technology. Micromeritics Instrument Corporation, Norcross (1997)

Winslow, D.N.: Advances in experimental techniques for mercury intrusion porosimetry. Surf. Colloid Sci. 13, 259-282 (1984)

Wilkinson, D., Willemsen, J.F.: Invasion percolation: a new form of percolation theory. J. Phys. A: Math. Gen. 16(14), 3365-3376 (1983)

Xiong, Q., Baychev, T.G., Jivkov, A.P.: Review of pore network modelling of porous media: experimental characterisations, network constructions and applications to reactive transport. J. Contam. Hydrol. 192, 101-117 (2016)

Publisher's Note Springer Nature remains neutral with regard to jurisdictional claims in published maps and institutional affiliations. 